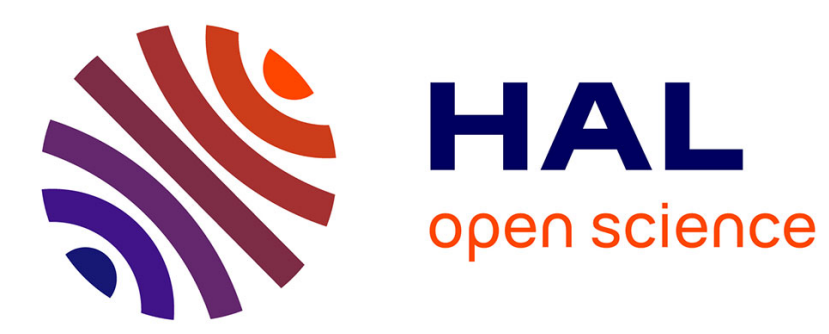

\title{
A Fast Multipole Method formulation for 3D elastodynamics in the frequency domain
}

\author{
Stéphanie Chaillat, Marc Bonnet, J. F. Semblat
}

\section{To cite this version:}

Stéphanie Chaillat, Marc Bonnet, J. F. Semblat. A Fast Multipole Method formulation for 3D elastodynamics in the frequency domain. Comptes Rendus Mécanique, 2007, 335, pp.709-714. 10.1016/j.crme.2007.07.001 . hal-00167345

\section{HAL Id: hal-00167345 \\ https://hal.science/hal-00167345}

Submitted on 28 Aug 2007

HAL is a multi-disciplinary open access archive for the deposit and dissemination of scientific research documents, whether they are published or not. The documents may come from teaching and research institutions in France or abroad, or from public or private research centers.
L'archive ouverte pluridisciplinaire HAL, est destinée au dépôt et à la diffusion de documents scientifiques de niveau recherche, publiés ou non, émanant des établissements d'enseignement et de recherche français ou étrangers, des laboratoires publics ou privés. 


\title{
A Fast Multipole Method formulation for 3D elastodynamics in the frequency domain
}

\author{
Stéphanie CHAILLAT ${ }^{\mathrm{a}, \mathrm{b}}$, Marc BONNET $^{\mathrm{a}}$ Jean-François SEMBLAT $^{\mathrm{b}}$ \\ ${ }^{\mathrm{a}} \mathrm{LMS}$, CNRS UMR 7649, Ecole Polytechnique, 91128 Palaiseau Cedex, France \\ ${ }^{\mathrm{b}}$ LCPC, 58 bd Lefebvre, 75732 Paris Cedex 15, France \\ Received $* * * * *$; accepted after revision +++++ \\ Presented by $\mathrm{xxxxx}$
}

\begin{abstract}
The solution of the elastodynamic equations using boundary element methods (BEMs) gives rise to fully-populated matrix equations. Earlier investigations on the Helmholtz and Maxwell equations have established that the Fast Multipole (FM) method reduces the complexity of a BEM solution to $N \log _{2} N$ per GMRES iteration. The present Note address the extension of the FM-BEM strategy to 3D elastodynamics in the frequency domain. Its efficiency and accuracy are demonstrated on numerical examples involving up to $N=O\left(10^{6}\right)$ nodal unknowns. To cite this article: S. Chaillat, M. Bonnet, J.F. Semblat, C. R. Mecanique 335 (2007).
\end{abstract}

\section{Résumé}

Méthode Multipôle Rapide pour l'élastodynamique 3D en domaine fréquentiel. La résolution des équations de l'élastodynamique par la méthode des éléments de frontière (BEM) conduit à un système linéaire plein. Des travaux récents sur les équations de Helmholtz et Maxwell ont établi la capacité de la méthode multipôle rapide $(\mathrm{FM})$ à réduire la complexité de la BEM à $N \log _{2} N$ par itération d'un solveur de type GMRES. Cette Note présente l'extension de l'approche FM-BEM à l'élastodynamique 3D dans le domaine fréquentiel. La précision et l'efficacité de la méthode sont illustrées sur des exemples numériques mobilisant jusqu'à $N=O\left(10^{6}\right)$ inconnues nodales. Pour citer cet article : S. Chaillat, M. Bonnet, J.F. Semblat, C. R. Mecanique 335 (2007).

Key words: computational solid mechanics ; boundary element method ; fast multipole method ; 3D elastodynamics Mots-clés : mécanique des solides numérique; éléments de frontière; méthode multipôle rapide; élastodynamique 3D

Email addresses: chaillat@lms.polytechnique.fr (Stéphanie CHAILLAT), bonnet@lms.polytechnique.fr (Marc BONNET), semblat@lcpc.fr (Jean-François SEMBLAT). 


\section{Version française abrégée}

La méthode des éléments de frontière, ou BEM [1], conduit dans sa forme classique à un système linéaire dont la matrice est pleine. Cela nuit grandement à son application aux modèles BEM à nombre $N$ de degrés de liberté (DDLs) grand $\left(N>O\left(10^{4}\right)\right)$ nécessaires aux calculs tridimensionnels réalistes. Les solveurs directs, de complexité $O\left(N^{3}\right)$ en temps et $O\left(N^{2}\right)$ en mémoire, deviennent inutilisables, et il faut recourir à des solveurs itératifs, de complexité $O\left(N^{2}\right)$ par itération. Pour éviter le stockage en mémoire de la matrice pleine, un réassemblage complet à chaque itération, très coûteux en intégrations numériques, est nécessaire. De plus, pour les analyses élastodynamiques en domaine fréquentiel objet de cette Note, $N$ est proportionnel au carré de la fréquence, ce qui limite le domaine des fréquences accessibles à la BEM.

L'accélération de l'évaluation des opérateurs intégraux, étape essentielle du calcul d'un produit matricevecteur utilisé par le solveur itératif (GMRES dans notre cas), permet de diminuer le temps de calcul d'une itération ainsi que les besoins en stockage, et d'élargir les capacités de la BEM. Une telle accélération est notamment permise par recours à la méthode multipôle rapide (Fast Multipole Method ou FMM). Initialement développée en électrostatique [2], la FMM est actuellement appliquée dans de nombreux domaines des sciences de l'ingénieur [3]. Cette Note a pour objet l'extension de la FMM à l'élastodynamique tridimensionnelle.

La BEM pour l'élastodynamique consiste à exploiter la formule de représentation intégrale (1) et l'équation intégrale (4). Les tenseurs de Green élastodynamiques (2) s'exprimant à l'aide de la solution élémentaire (3) de l'équation de Helmholtz (pour les nombres d'onde de compression et de cisaillement), ils peuvent être reformulés en termes du développement en série de multipôles (5), déjà utilisé en électromagnétisme $[4,8]$. La séparation des variables $\boldsymbol{x}$ et $\boldsymbol{y}$ ainsi introduite permet la réutilisation d'intégrations par rapport à $\boldsymbol{y}$ dans (1) ou (4) quand le point de collocation $\boldsymbol{x}$ change, ce qui est impossible en BEM classique. Le développement (5) permet par ailleurs le regroupement de calculs d'influences entre « paquets » de points $\boldsymbol{x}$ et $\boldsymbol{y}$ spatialement séparés, définis dans la procédure numérique au moyen d'un découpage en cellules cubiques de la région d'espace contenant la frontière du domaine analysé. Un découpage non récursif (une seule taille de cellule) mène à la FMM mono-niveau, qui permet le calcul du produit matrice-vecteur en $O\left(N^{3 / 2}\right)$ opérations. Un perfectionnement supplémentaire consiste à effectuer le découpage en cellules de façon récursive (cellules découpées en sous-cellules, sur plusieurs niveaux); la FMM multi-niveaux ainsi définie réduit la complexité précédente à $O\left(N \log _{2} N\right)$ opérations (Figure 1). Enfin, le coût mémoire est également réduit à $O\left(N \log _{2} N\right)$ (Figure 1) par le fait que seules les contributions correspondant aux interactions proches sont assemblées et conservées.

Des tests sur un cas à solution exacte connue valident la méthode et montrent sa précision (Table 1). En particulier, l'erreur introduite par la FMM par rapport à la BEM classique (troncature de la série et intégration numérique sur la sphère unité dans (5)) n'ont pas d'incidence pratique sur la qualité du résultat. Cette approche permet ainsi, par exemple dans le domaine de la sismologie, de traiter des configurations plus réalistes et pour un domaine de fréquences plus grand (calcul d'effet de site sismique, figure 3).

\section{Introduction}

The main advantage of the boundary element method (BEM [1]) is that only the domain boundaries (and possibly interfaces) are discretized, leading to a reduction of the number of degrees of freedom (DOFs). However, standard BEM algorithms give rise to fully-populated linear systems of equations, making it very expensive in terms of CPU times $\left(O\left(N^{2}\right)\right.$ per iteration using an iterative solver such as GMRES) and memory requirements for BEM models featuring large numbers $N$ of DOFs. 
In other areas such as computational electromagnetism or acoustics, considerable improvements in the computing speed and memory efficiency of BEM algorithms have been achieved on the basis of the Fast Multipole Method (FMM) [3], with solution times down to $O\left(N \log _{2} N\right)$ per iteration. So far, very few studies (which include [5] for the frequency-domain case and [6] for the time-domain case) have been devoted to fast multipole-accelerated BEMs (FM-BEMs) in elastodynamics. The present Note improves on the methodology of [5] by incorporating recent advances of FMM implementations for Maxwell equations (e.g. [8]), which allow to run BEM models of much larger size.

\section{Boundary integral equation method}

Boundary integral representation. Let $\Omega$ denote the region of space occupied by an elastic solid with isotropic constitutive properties defined by $\mu$ (shear modulus), $\nu$ (Poisson's ratio) and $\rho$ (mass density). Assuming a time-harmonic motion with circular frequency $\omega$ and the absence of body forces, the displacement $\boldsymbol{u}$ is given at an interior point $\boldsymbol{x} \in \Omega$ by the following well-known representation formula [1]:

$$
u_{k}(\boldsymbol{x})=\int_{\partial \Omega}\left[t_{i}(\boldsymbol{y}) U_{i}^{k}(\boldsymbol{x}, \boldsymbol{y} ; \omega)-u_{i}(\boldsymbol{y}) T_{i}^{k}(\boldsymbol{x}, \boldsymbol{y} ; \omega)\right] \mathrm{d} S_{y} \quad(\boldsymbol{x} \in \Omega),
$$

where $\boldsymbol{t}$ is the traction vector on the boundary $\partial \Omega$, and $U_{i}^{k}(\boldsymbol{x}, \boldsymbol{y} ; \omega)$ and $T_{i}^{k}(\boldsymbol{x}, \boldsymbol{y} ; \omega)$ denote the $i$-th components of the elastodynamic fundamental solution, i.e. of the displacement and traction, respectively, generated at $\boldsymbol{y} \in \mathbb{R}^{3}$ by a unit point force applied at $\boldsymbol{x} \in \mathbb{R}^{3}$ along the direction $k$, given by [7]:

$$
\begin{aligned}
U_{i}^{k}(\boldsymbol{x}, \boldsymbol{y} ; \omega) & =\frac{1}{4 \pi k_{S}^{2} \mu}\left(\left(\delta_{q s} \delta_{i k}-\delta_{q k} \delta_{i s}\right) \frac{\partial}{\partial x_{q}} \frac{\partial}{\partial y_{s}} G_{S}(|\boldsymbol{y}-\boldsymbol{x}|)+\frac{\partial}{\partial x_{i}} \frac{\partial}{\partial y_{k}} G_{P}(|\boldsymbol{y}-\boldsymbol{x}|)\right), \\
T_{i}^{k}(\boldsymbol{x}, \boldsymbol{y} ; \omega) & =\mu\left[\frac{2 \nu}{1-2 \nu} \delta_{i j} \delta_{k \ell}+\delta_{i k} \delta_{j \ell}+\delta_{j k} \delta_{i \ell}\right] \frac{\partial}{\partial y_{\ell}} U_{h}^{k}(\boldsymbol{x}, \boldsymbol{y} ; \omega) n_{j}(\boldsymbol{y}),
\end{aligned}
$$

in which $G_{\alpha}(|\boldsymbol{y}-\boldsymbol{x}|)(\alpha=S, P)$, defined by

$$
G_{\alpha}(|\boldsymbol{y}-\boldsymbol{x}|)=\frac{\exp \left(i k_{\alpha}|\boldsymbol{y}-\boldsymbol{x}|\right)}{|\boldsymbol{y}-\boldsymbol{x}|} \quad k_{S}^{2}=\frac{\rho \omega^{2}}{\mu}, \quad k_{P}^{2}=\frac{1-2 \nu}{2(1-\nu)} k_{S}^{2},
$$

is the free-space Green's function for the Helmholtz equation with wavenumber $k_{\alpha}$ corresponding to either $P$ or $S$ elastic waves, and $\boldsymbol{n}(\boldsymbol{y})$ is the unit normal to $\partial \Omega$ directed outwards of $\Omega$.

Boundary integral formulation. When $\boldsymbol{x} \in \partial \Omega$, a singularity occurs in $\boldsymbol{y}=\boldsymbol{x}$. With the help of a well-documented limiting process, the integral representation (1) yields the integral equation:

$$
c_{i k}(\boldsymbol{x}) u_{i}(\boldsymbol{x})=\mathrm{PV} \int_{\partial \Omega} t_{i}(\boldsymbol{y}) U_{i}^{k}(\boldsymbol{x}, \boldsymbol{y} ; \omega) \mathrm{d} S_{y}-\int_{\partial \Omega} u_{i}(\boldsymbol{y}) T_{i}^{k}(\boldsymbol{x}, \boldsymbol{y} ; \omega) \mathrm{d} S_{y} \quad(\boldsymbol{x} \in \partial \Omega),
$$

where ' $\mathrm{PV}$ ' indicates a Cauchy principal value $(\mathrm{CPV})$ singular integral and the free-term $c_{i k}(\boldsymbol{x})$ is equal to $0.5 \delta_{i k}$ in the usual case where $\partial \Omega$ is smooth at $\boldsymbol{x}$. Equation (4) may be recast into alternative, equivalent regularized forms which are free of CPV integrals [1].

\section{Fast Multipole Method: principle}

One-level algorithm. The goal of the FMM is to speed up the matrix-vector product computation required for each iteration of the iterative solver applied to the BEM-discretized equations arising from (4). Moreover, the governing matrix is never explicitly formed, which leads to a storage requirement well below 
the $O\left(N^{2}\right)$ memory required for holding the whole BEM matrix. The FM-accelerated BEM, described next, therefore achieves substantial savings in both CPU time and memory.

In general terms, the main idea behind the FMM is to reformulate the fundamental solutions in terms of products of functions of $\boldsymbol{x}$ and of $\boldsymbol{y}$. This allows to reuse integrations with respect to $\boldsymbol{y}$ when the collocation point $\boldsymbol{x}$ is changed, a strategy which is not feasible in the traditional BEM, based on expressions (2). The latter are seen to be linear combinations of the Green's function for the Helmholtz equation (3), for which such a reformulation is known from earlier works, e.g. [3,4,8]. The position vector $\boldsymbol{r}=\boldsymbol{y}-\boldsymbol{x}$ can be decomposed as $\boldsymbol{r}=\left(\boldsymbol{x}_{\mathbf{0}}-\boldsymbol{x}\right)+\left(\boldsymbol{y}_{\mathbf{0}}-\boldsymbol{x}_{\mathbf{0}}\right)-\left(\boldsymbol{y}_{\mathbf{0}}-\boldsymbol{y}\right)=\tilde{\boldsymbol{x}}+\boldsymbol{r}_{\mathbf{0}}-\tilde{\boldsymbol{y}}$, where $\boldsymbol{x}_{\mathbf{0}}$ and $\boldsymbol{y}_{\mathbf{0}}$ are two poles. Application of the Gegenbauer addition theorem [8] permits to write the Helmholtz Green's function as:

$$
\frac{\exp (i k|\boldsymbol{y}-\boldsymbol{x}|)}{|\boldsymbol{y}-\boldsymbol{x}|}=\frac{i k}{4 \pi} \lim _{L \rightarrow+\infty} \int_{\tilde{\boldsymbol{s}} \in S} e^{i k \tilde{\boldsymbol{s}} \cdot \tilde{\boldsymbol{x}}} \mathcal{G}_{L}\left(\tilde{\boldsymbol{s}} ; \boldsymbol{r}_{\mathbf{0}}\right) e^{-i k \tilde{\boldsymbol{s}} \cdot \tilde{\boldsymbol{y}}} d \tilde{\boldsymbol{s}}
$$

where $S$ is the unit sphere of $\mathbb{R}^{3}$ and the transfer function $\mathcal{G}_{L}\left(\tilde{\boldsymbol{s}} ; \boldsymbol{r}_{\mathbf{0}}\right)$ is defined by:

$$
\mathcal{G}_{L}\left(\tilde{\boldsymbol{s}} ; \boldsymbol{r}_{\mathbf{0}}\right)=\sum_{0 \leq l \leq L}(2 l+1) i^{\ell} h_{\ell}^{(1)}\left(k\left|\boldsymbol{r}_{\mathbf{0}}\right|\right) P_{\ell}\left(\cos \left(\tilde{\boldsymbol{s}}, \boldsymbol{r}_{\mathbf{0}}\right)\right)
$$

in terms of the Legendre polynomials $P_{\ell}$ and the spherical Hankel functions of the first kind $h_{\ell}^{(1)}$. Then, the elastodynamic fundamental solution (2) is easily seen to admit representations of the form (5) with $\mathcal{G}_{L}$ replaced with suitably-defined (tensor) transfer functions $\mathcal{U}_{i, L}^{k}$ and $\mathcal{T}_{i, L}^{k}$.

Formula (5) with $L=O\left(k_{S}\left|\boldsymbol{r}-\boldsymbol{r}_{\mathbf{0}}\right|\right)$ is suitably accurate whenever $\left|\boldsymbol{r}-\boldsymbol{r}_{\mathbf{0}}\right| /\left|\boldsymbol{r}_{\mathbf{0}}\right| \leq 2 / \sqrt{5}$ [8]. Guided by this consideration, a $3 \mathrm{D}$ cubic grid of linear spacing $d$ embedding the whole boundary $\partial \Omega$ is introduced. The above condition is then automatically satisfied whenever $\boldsymbol{x}$ and $\boldsymbol{y}$ belong to non-adjacent cubic cells, the poles $\boldsymbol{x}_{\mathbf{0}}$ and $\boldsymbol{y}_{\mathbf{0}}$ being chosen as the cell centers, while it may be violated otherwise. The one-level FMM basically consists of using decomposition (5) whenever $\boldsymbol{x}$ and $\boldsymbol{y}$ belong to non-adjacent cubic cells. A typical contribution of this kind to the residual of (1) or (4) is of the form:

$$
I_{k}(\boldsymbol{x})=\int_{\partial \Omega \cap \mathcal{C}_{y}} t_{i}(\boldsymbol{y}) U_{i}^{k}(\boldsymbol{x}, \boldsymbol{y}, \omega) d S_{y} \quad\left(\boldsymbol{x} \in \mathcal{C}_{x}\right)
$$

where $\mathcal{C}_{x}$ and $\mathcal{C}_{y}$ denote cubic cells with respective centers $\boldsymbol{x}_{\mathbf{0}}$ and $\boldsymbol{y}_{\mathbf{0}}$ (which are used as poles in decomposition (5)) and $\boldsymbol{t}$ is associated to a solution candidate. On inserting (5), for a well-chosen finite truncation value $L$, into $(2), I_{k}(\boldsymbol{x})$ is evaluated by means of a three-step computational procedure which is summarized by:

$$
\begin{aligned}
\mathcal{R}_{i}\left(\tilde{\boldsymbol{s}}_{q} ; \boldsymbol{y}_{\mathbf{0}}\right) & =\int_{\partial \Omega \cap \mathcal{C}_{y}} t_{i}\left(\boldsymbol{y}_{\mathbf{0}}+\tilde{\boldsymbol{y}}\right) e^{-i k \tilde{\boldsymbol{s}}_{q} \cdot \tilde{\boldsymbol{y}}} d S_{\tilde{y}} & & \text { computation of multipole moments } \\
\mathcal{L}_{k}\left(\tilde{\boldsymbol{s}}_{q} ; \boldsymbol{x}_{\mathbf{0}}\right) & =\mathcal{U}_{i, L}^{k}\left(\tilde{\boldsymbol{s}}_{q} ; \boldsymbol{r}_{\mathbf{0}}\right) \mathcal{R}_{i}\left(\tilde{\boldsymbol{s}}_{q} ; \boldsymbol{y}_{\mathbf{0}}\right) & & \text { transfer } \\
I_{k}(\boldsymbol{x}) & \approx \sum_{q} w_{q} \frac{i k}{4 \pi} e^{i k \tilde{\boldsymbol{s}}_{q} \cdot \tilde{\boldsymbol{x}}_{k}} \mathcal{L}_{k}\left(\tilde{\boldsymbol{s}}_{q} ; \boldsymbol{x}_{\mathbf{0}}\right) & & \text { local expansion }
\end{aligned}
$$

where $\tilde{\boldsymbol{s}}_{q}$ and $w_{q}$ are quadrature points and weights for the integration over the unit sphere. When cells $\mathcal{C}_{x}$ and $\mathcal{C}_{y}$ are adjacent, traditional BEM evaluation methods based on expressions (2) and (3) are used.

The one-level fast multipole method in the elastodynamics case has an optimal complexity of $O\left(N^{3 / 2}\right)$. This complexity is achieved by using $O\left(N^{3 / 4}\right)$ cells. It is already more efficient than the classical BEM, but the multi-level algorithm provides further acceleration.

Multi-level algorithm. To improve further the computational efficiency of the FM-BEM, standard (i.e. non-FMM) calculations must be confined to the smallest possible spatial regions while retaining the 
advantage of clustering the computation of influence terms into non-adjacent large groups whenever possible. This idea is carried out by subdividing cubic cells into eight smaller cubic cells. New pairs of non-adjacent smaller cells, to which multipole expansions are applicable, are thus obtained from the subdivision of pairs of adjacent cells. In this way, the non-FMM computations are reduced to influence evaluations between the pairs of adjacent cells at the deepest subdivision level, i.e. to a smaller proportion of all influence computations than in the one-level FMM.

Previous studies for the Maxwell equations (e.g. [8]) have established a crucial practical consideration: for a given desired accuracy, both the truncation parameter $L$ in the transfer function (6) and the number of quadrature points $\tilde{\boldsymbol{s}}_{q}$ in (7)-(9) depend on the subdivision level, and in fact increase with $k_{\alpha} d$ (the cell-size-to-wavelength ratio). The present implementation incorporates these findings, whereas that of [5] does not. Accounting for these effects, the theoretical complexity of the multi-level FMM is $O\left(N \log _{2} N\right)$ per iteration both for CPU time and memory (i.e. somewhat higher than the $O(N)$ complexity for static FM-BEM, where the truncation parameter in the FMM expansion is not level-dependent).

\section{Fast Multipole Method: accuracy and computational efficiency}

The examples below use piecewise-linear interpolation of displacements (3-noded triangular boundary elements) and have been run on the same single-processor PC (CPU frequency: $3.40 \mathrm{GHz}$, RAM: 3Go).

Spherical cavity under internal pressure. A spherical cavity of radius $R$ embedded in an elastic isotropic infinite medium (with $\nu=0.25$ ) is subjected to an internal uniform pressure $P$. This problem has a simple, spherically-symmetric, exact solution [7], against which numerically-computed solutions are compared using the relative root mean square solution error. The results of such comparisons, performed for several normalized frequencies $\eta_{P}=k_{P} R / \pi$ and various distances from the cavity wall $(R \leq r \leq 3 R)$ are presented in Table 1 . They indicate that the present FM-BEM is very accurate, even in the nearly-static case $\left(\eta_{P}=0.01\right)$ for which the accuracy of FMM expansions of the form (5) is known to deteriorate [8] (whereas the standard BEM does not [9]). Moreover, the accuracy is seen to deteriorate, as expected, for mesh densities below about eight nodes per $S$ wavelength. In all subsequent results, the meshes are designed so as to feature at least 10 nodes per $S$ wavelength. Finally, the numerical experiments corroborate previously mentioned theoretical complexity estimates for standard BEM, one-level FMM and multi-level FMM (Figure 1). The largest problem size for this complexity study is $N \approx 10^{6}$, while the examples of [5] used $N \leq 2.510^{4}$.

\begin{tabular}{|c|c|c|c|c|}
\hline$\eta_{P}$ & 0.01 & 0.50 & 1.00 & 2.00 \\
\hline nb nodes $/ \lambda_{S}$ & 800 & 16 & 8 & 4 \\
\hline cavity wall $r=R$ & 0.018 & 0.006 & 0.006 & 0.021 \\
\hline domain $R<r \leq 3 R$ & 0.017 & 0.006 & 0.008 & 0.031 \\
\hline \\
Table 1 cavity and in the domain \\
RMS solution error on the can
\end{tabular}

Diffraction of an incident $P$ plane wave by a semi-spherical canyon. The diffraction of verticallytravelling P-waves by a semi-spherical canyon of radius $R$ in an elastic half space (with $\nu=0.25$ ) is now considered. This problem constitutes an idealized configuration for studying topographic site effects in seismology. The semi-spherical surface of the canyon and the surrounding portion of free surface lying inside a disk of radius $D>R$ are discretized using boundary elements. This problem has been previously 

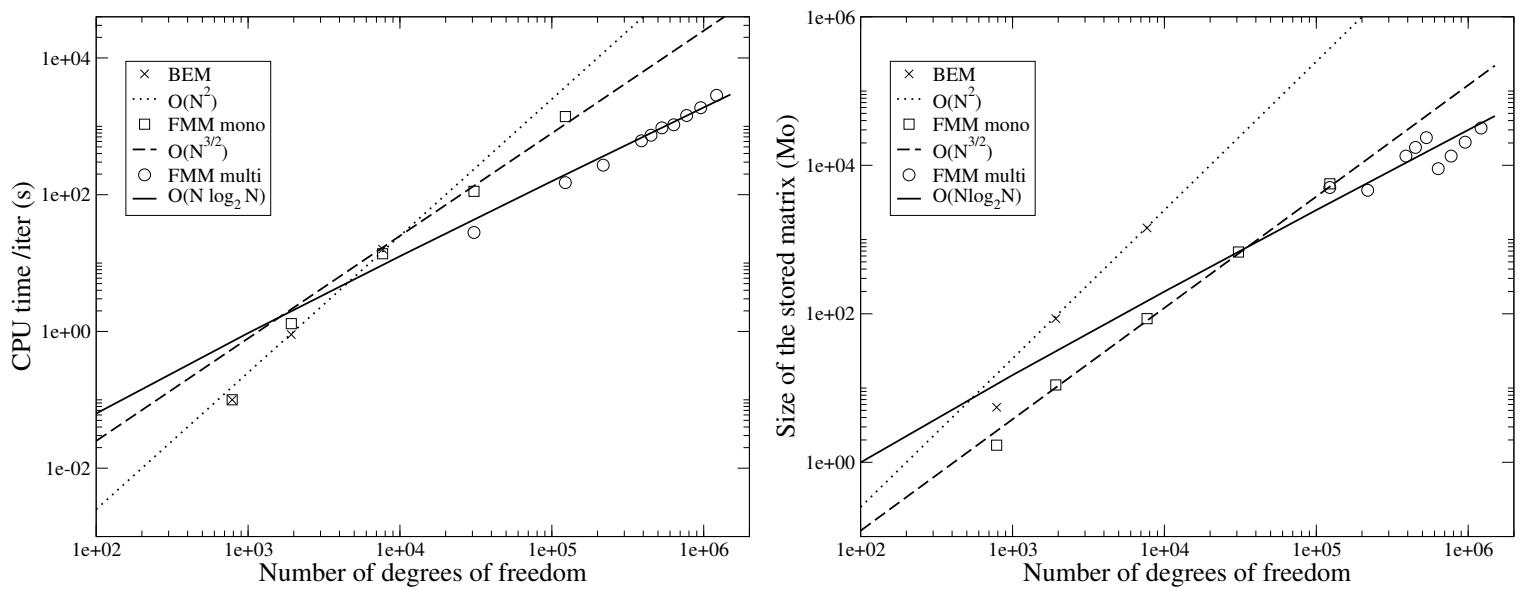

Figure 1. Complexity of the standard BEM, one-level FMM and multi-level FMM (left: CPU time, right: memory)

investigated in [10] using a (semi-analytical) series expansion method. The moduli of the vertical and horizontal displacements, computed using the present FMM (7794 nodes, 7 GMRES iterations, 24s of CPU time per iteration), are compared in Figure 2 to corresponding results from [10], for $\eta_{P}=0.25$ and with $D=3 R$. A good agreement between both approaches is observed, the main discrepancies occuring at points such that $r / R \geq 2$ (i.e. close to the truncation radius $D=3 R$ ) due to the differences in handling the truncation in both studies. The case $\eta_{P}=5$ (Figure 3$)$, whose size $(N=287946)$ is well beyond the capabilities of standard BEM, has been solved using the present FMM (86 GMRES iterations, $5 \mathrm{mn}$ CPU time per iteration).

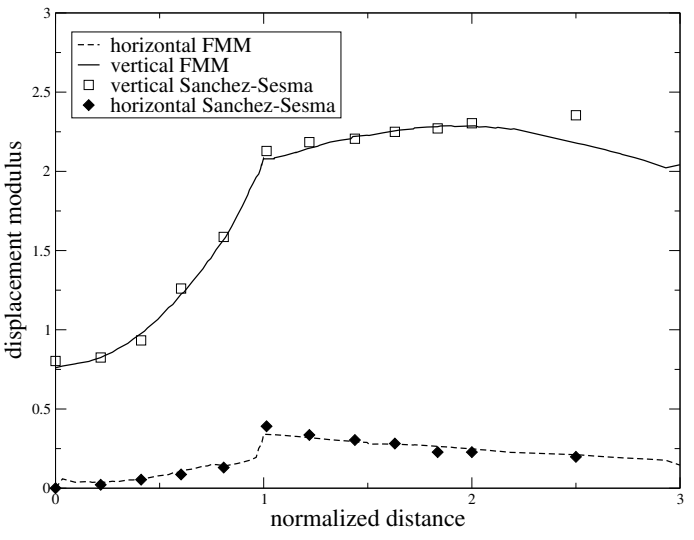

Figure 2. Comparison of the numerical FMM solution and the results of $[10]$ for $\eta_{P}=0.25$

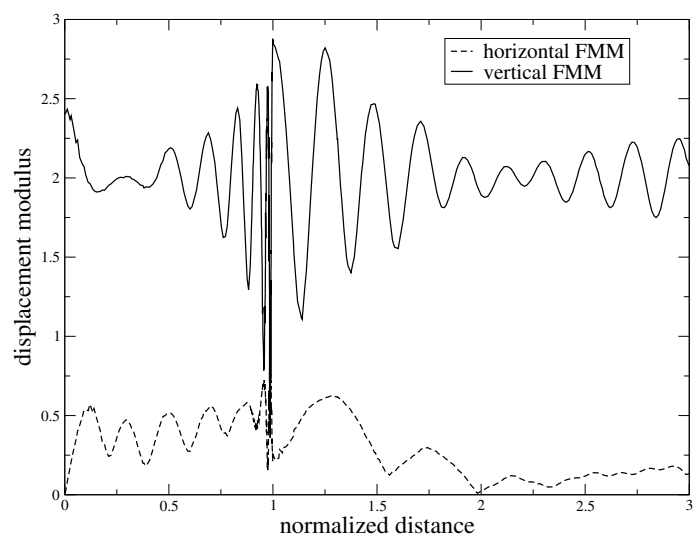

Figure 3. Numerical FMM solution for $\eta_{P}=5$

\section{Conclusions}

In this Note, the Fast Multipole Method has been succesfully extended to $3 D$ elastodynamics in the frequency domain. Combined with the BEM formulation, it permits to reduce the computational burden, in both CPU time and memory requirements, for the analysis of wave propagation (e. g. seismic), and 
allows to run BEM models of size $N=O\left(10^{6}\right)$ on an ordinary PC. Comparisons with analytical or previously published numerical results show the accuracy of the present method. Moreover, observed CPU times are found to be consistent with known theoretical complexity estimates.

\section{References}

[1] M. Bonnet, Boundary integral equation method for solids and fluids, Wiley, 1999.

[2] L. Greengard, V. Rokhlin, A fast algorithm for particle simulations, J. Comp. Phys. 73(1987) 325-348.

[3] N. Nishimura, Fast multipole accelerated boundary integral equation methods, Appl. Mech. Rev. 55(2002) 299-324.

[4] J.M. Song, C. C. Lu, W. C. Chew, Multilevel fast multipole algorithm for electromagnetic scattering, IEEE Antennas and Propagation 45(1997) 1488-1493.

[5] H. Fujiwara, The fast multipole method for solving integral equations of three-dimensional topography and basin problems, Geophysical Journal International 140(2000) 198-210.

[6] T. Takahashi, N. Nishimura, S. Kobayashi S, A fast BIEM for three-dimensional elastodynamics in time domain. Engineering Analysis with Boundary Elements 27(2003) 491-506.

[7] A.C. Eringen, E.S. Suhubi, Elastodynamics, II-linear theory, Academic Press, 1975.

[8] E. Darve, The fast multipole method: numerical implementation. Journal of Computational Physics 160(2000) 195-240.

[9] P. Dangla, J. F. Semblat, H. Xiao, N. Delépine, A simple and efficient regularization method for 3d bem : application to frequency-domain elastodynamics, Bulletin of the Seismological Society of America 95(2005) 1916-1927.

[10] F. J. Sánchez-Sesma, Diffraction of elastic waves by 3D surface irregularities, Bulletin of the Seismological Society of America 73(1983) 1621-1636. 